den Arbeitsmarkt zu vermindern. ${ }^{18}$ Diese Vorschläge sind problematisch. Bereits jetzt sind die Erwerbsquoten von alleinerziehenden Müttern höher als die von verheirateten Müttern, aber das Erwerbseinkommen ist häufig relativ niedrig. Insbesondere für erwerbstätige Alleinerziehende sind ergänzende Sozialleistungen sinnvoll; der Kinderzuschlag ist hier bisher nicht zielführend. Außerdem sollten Beiträge für eine ausreichende Altersvorsorge in höherem Umfang von Erwerbseinkommen abzugsfähig sein als bisher. Vermittlung in geringfü- gige Beschäftigungsverhältnisse eröffnet jedoch keine sinnvolle langfristige Perspektive, ist in der Praxis der Jobcenter für Mütter junger Kinder jedoch häufig. Deshalb wäre es empfehlenswert, für ,Berufsrückkehrerinnen' wieder Rechtsansprüche auf Qualifizierungs- und Umschulungsmaßnahmen einzuführen, die jedoch durch Hartz III eingeschränkt und durch Ermessensleistungen ersetzt wurden.

18 So etwa Eichenhofer (FN 8), S. B 38.

\title{
Finanzmarktkrise, Föderalismusreform und die Folgen - ein Ausblick
}

Bund, Länder und Gemeinden müssen laut der Steuerschätzung vom Mai 2009 bis 2013 mit über 300 Milliarden Euro weniger auskommen als noch im November 2008 angenommen. Allein für 2009 wird erwartet, dass die Steuereinnahmen um 45 Milliarden Euro niedriger ausfallen. Gleichzeitig steigen die Kosten: Höhere Arbeitslosigkeit führt nicht nur dazu, dass weniger Menschen in die Sozialversicherung einzahlen; es werden auch mehr Ansprüche geltend gemacht. Das Kurzarbeitergeld belastet den Haushalt der Bundesagentur für Arbeit (BA) zusätzlich. Die BA rechnet damit, dass sie in diesem Jahr 3,4 Milliarden Euro für die Kurzarbeit benötigen wird, sagte BA-Vorstandsmitglied Raimund Becker im Juni der „Süddeutschen Zeitung“. Dies ist über eine Milliarde mehr als geplant. Es ist somit absehbar, dass die Bundesagentur höhere finanzielle Unterstützung aus dem Bundeshalt benötigt. Hinzu kommen die Maßnahmen der Konjunkturpakete auf Bundes- und Länderebene, die ebenfalls finanziert werden müssen, der „Schutzschild“ für die Banken und andere Stabilisierungsmaßnahmen.

Zudem hat die Kommission von Bundesrat und Bundestag zur Modernisierung der Bund-LänderFinanzbeziehungen (Föderalismuskommission II) im März dieses Jahres die Einführung einer Schuldenbegrenzungsregelung beschlossen. Diese wurde mit dem anschließenden Gesetzgebungsverfahren, das am 12. Juni 2009 im Bundesrat abgeschlossen worden ist, im Grundgesetz verankert. Die Schuldengrenze tritt für den Bund 2016, für die Länder 2020 in Kraft. Konnten Bund und Länder bisher in der Höhe, in der sie Investitionen tätigten, Kredite aufnehmen, ist dieser Weg dann weitgehend verschlossen. Die neue Regelung begrenzt die Möglichkeit einer strukturellen Neuverschuldung - also einer solchen, die nicht zurückgeführt werden muss - für den Bund auf 0,35 Prozent des Bruttoinlandsproduktes, was derzeit etwa neun Milliarden Euro im Jahr entspricht. Für die Länder ist eine strukturelle Verschuldung dann gar nicht mehr möglich. Zulässig ist ab Inkrafttreten für die Länder nur noch eine sogenannte konjunkturelle Verschuldung, mit der auf konjunkturelle Schwankungen reagiert werden kann. Diese hat aber nahe der Null zu bleiben und muss innerhalb eines angemessenen Zeitraumes zurückgeführt werden. In Notsituationen (Naturkatastrophen, aber auch einer der jetzigen vergleichbaren Finanzmarktkrise) ist eine Verschuldung weiterhin erlaubt, jedoch gekoppelt an einen Tilgungsplan - auch hier also mit Rückführungsverpflichtung. Konjunkturelle Verschuldungsmöglichkeiten und Notfallverschuldung sind auch für den Bund möglich.

Auch wenn bis zum eigentlichen Inkrafttreten noch einige Jahre vergehen - Auswirkungen sind bereits in naher Zukunft zu erwarten, denn bis 2016 bzw. 2020 müssen der Bund und die Länder erreichen, dass sie diese Vorgaben einhalten können. Es ist also davon auszugehen, dass sie in den nächsten Jahren finanzpolitisch einen harten Konsolidierungskurs fahren, dies bei einer voraussichtlich auch noch mittelfristig schwachen Wirtschaftsentwicklung mit entsprechend geringen Steuereinnahmen. Dieser Weg ist bei einer Neuverschuldung, die Bundesfinanzminister Peer Steinbrück für dieses Jahr mit 80 Milliarden Euro für den Bundeshaushalt beziffert, noch sehr lang, nicht zuletzt, weil man dies mit den mittelfristig niedrigeren Steuereinnahmen zusammen sehen muss.

Gerade in Zeiten leerer Kassen ist eine Diskussion über eine weitere Privatisierung von Risiken sei es bei der Rente, der Krankenversicherung oder

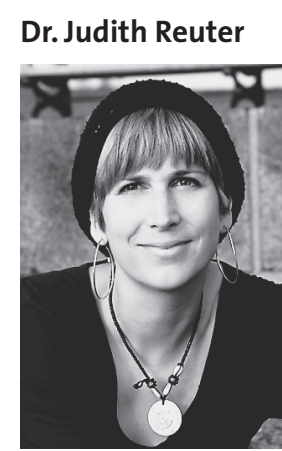

Mitglied der Kommission Recht der sozialen Sicherung, Familienlastenausgleich des djb; Referentin für Bund-Länder-Finanzbeziehungen und Föderalismusreform, Senatskanzlei, Hamburg 
der Pflegeversicherung - zu befürchten. In diesen Zeiten ist es umso wichtiger, das, was in den vergangenen 60 Jahren beim Abbau der Diskriminierung nach dem Geschlecht erreicht worden ist, zu verteidigen. Erreichte Erweiterungen gesetzlicher Leistungen sollen erhalten bleiben. Eine weitere Erosion der Sozialversicherungssysteme durch die Streichung der verbliebenen solidarisch umverteilenden Elemente gilt es zu verhindern. Dass Wettbewerb und Markt nicht der einzig gültige Maßstab sein kann, haben die letzten Monate deutlich gezeigt.

Zum anderen zeigt die Finanzmarktkrise deutlich, dass sich Armutsrisiken auch verwirklichen und Frauen hiervon ganz besonders getroffen sind: prekäre Beschäftigungsverhältnisse werden weiter zunehmen - betroffen sind zumeist Frauen. Unterbrochene Erwerbsbiografien führen zu niedrigen Rentenansprüchen - dies gilt weiterhin vor allem für Frauen. Hinzu kommt, dass das Ehegattensplitting und die Zusammenveranlagung von Eheleuten immer noch Frauen zur Aufgabe ihrer
Erwerbstätigkeit motivieren. Auch hieraus ergeben sich im Falle einer Scheidung Armutsrisiken. Berufsrückkehrerinnen werden es auf dem Arbeitsmarkt ohne entsprechende Qualifizierung noch schwerer haben. Die Anrechnung des Partnereinkommens beim SGB II führt zu einem Verlust von Leistungsansprüchen, der einen Wiedereinstieg in den Arbeitsmarkt weiter erschwert.

Ein Augenmerk sollte in näherer Zukunft daher insbesondere darauf liegen, wie durch strukturelle Veränderungen in Zukunft Frauen eigenständig und individuell abgesichert sein können, um Armut zu vermeiden und Teilhabe zu ermöglichen. Das djb-Rentenmodell ist ein Beispiel für einen solchen Vorschlag für eine strukturelle Veränderung sozialer Sicherungssysteme. Hier gilt es weiterzudenken.

Gleichstellungspolitik ist kein Luxus und kein Thema für gute Zeiten. Das Gebot des Art. 3 Abs. 2 GG ist keines, das man sich leisten können muss. Es gilt immer.

\section{Gender Pay Gap mit neuen Instrumenten überwinden}

Prof. Dr. Sibylle Raasch

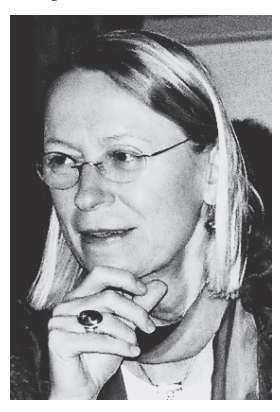

Mitglied der Kommission Arbeits-, Gleichstellungs- und Wirtschaftsrecht des djb; Hochschullehrerin, z. Zt. Universität Wien
Der Gender Pay Gap, also die Entgeltdifferenz zwischen Frauen und Männern, betrug im Jahr 2007 nach Angaben des Statistischen Bundesamtes ${ }^{1}$ in Deutschland 23 Prozent, sogar 24 Prozent in den alten Bundesländern, aber nur 6 Prozent in den neuen Bundesländern. Die Bundesrepublik ist in Sachen Entgeltgleichheit auch nicht auf einem „guten Weg“. Der Verdienstabstand wächst tendenziell weiter. Nur im Bereich Verkehr und Nachrichtenübermittlung hat sich der Abstand der durchschnittlichen Bruttostundenverdienste 2006 bis 2007 verringert - um gerade einmal ein Prozent. $^{2}$

Dieser Gender Pay Gap ist ein Ergebnis des Zusammenwirkens verschiedener Faktoren im Sinne struktureller Frauenbenachteiligung. Hierzu zählen insbesondere: die fortbestehende Abwertung von Tätigkeiten und Verhaltensweisen, die mit Frauen und Weiblichkeit identifiziert werden; die Dominanz des Familienernährer-Zuverdienerinnen-Modells als Lebensform; das Verbleiben der Haus- und Familienarbeit vorrangig bei den Frauen, selbst wenn partnerschaftlicher gelebt werden soll; die geschlechtsspezifisch getrennten Arbeitsmärkte in der Gesamtwirtschaft und in den einzelnen Betrieben, wobei die Frauen weniger Bereiche mit schlechterer Vergütung und schlechteren Aufstiegschancen besetzen; die geringe Vereinbarkeit von Beruf und Familie mit der Folge, dass Frauen ihre Erwerbstätigkeit häufiger und länger unterbrechen als Männer und deswegen erhebliche Gehaltseinbußen hinneh- men müssen ${ }^{3}$ oder in Teilzeitarbeit ausweichen; die Diskriminierung von Teilzeitarbeit; die Diskriminierung von Frauen bei der Einstellung und beim beruflichen Aufstieg; die unverändert extreme Unterrepräsentanz von Frauen in Führungs- und Leitungspositionen; verschiedene Formen von Entgeltdiskriminierung. ${ }^{4}$

Der Gender Pay Gap muss also durch Maßnahmen in allen diesen Bereichen bekämpft werden. Die bessere Vereinbarkeit von Beruf und Familie ist hierbei nur ein Mosaiksteinchen, nicht die Lösung des Gesamtproblems, wie es Erklärungen der Bundesregierung aber häufig nahelegen. Auf ein gezieltes Vorgehen gegen direkte und mittelbare Entgeltdiskriminierung kann aus Frauensicht keinesfalls verzichtet werden.

\section{Verbesserungen auf freiwilliger Basis nicht zu er- warten}

Die Vereinbarung zwischen der Bundesregierung und den Spitzenverbänden der deutschen Wirtschaft zur Förderung der Chancengleichheit von Frauen und Männern aus dem Jahr 2001 setzt auf

1 Vgl. Statistisches Bundesamt, PM Nr. 427 vom 14.11.2008.

2 Vgl. Statistisches Bundesamt a.a.O.

3 Vgl. neuste Berechnungen von Boll, Christina: Lohneinbußen durch geburtsbedingte Erwerbsunterbrechungen..., HWWI Research Paper 1-19, Hamburg Januar 2009.

4 Vgl. Droßard, Ralf: Verdienstabstand zwischen Frauen und Männern, Destasis, 26.8.2008. 